\title{
Non-Exposure Endoscopic-Laparoscopic Cooperative Surgery for Stomach Tumors
}

\author{
Hyo-Jung $\mathrm{Oh}^{1,2}$ and Chan-Young Kim ${ }^{2,3}$ \\ ${ }^{1}$ Graduate School of Archives and Records Management, Chonbuk National University, Jeonju, ${ }^{2}$ Division of Gastrointestinal Surgery, Department \\ of Surgery, Chonbuk National University Medical School, Jeonju, ${ }^{3}$ Institute of Medical Information Convergence Research in CBNU, Jeonju, \\ Korea
}

See "Non-Exposure Endoscopic-Laparoscopic Cooperative Surgery for Stomach Tumors: First Experience from the Czech Republic" by Jan Hajer, Lukáš Havlůj, Adam Whitley, et al., on page 167-173.

Hajer et al. ${ }^{1}$ reported the first experience in Czech Republic of using non-exposure endoscopic-laparoscopic cooperative surgery for stomach tumors. All 12 patients were successfully treated. The tumors were gastrointestinal stromal tumor (GIST), early gastric cancer, neuroendocrine tumor, Vanek's tumor, lipoma, and ectopic pancreatic tissue. The authors used two representative methods of the non-exposure technique as follows: "combination of laparoscopic and endoscopic approaches to non-exposure technique" (CLEAN-NET) ${ }^{2}$ and "non-exposure endoscopic wall-inversion surgery" (NEWS). ${ }^{3}$ The removal of benign tumors of the stomach is generally performed using laparoscopic wedge resection; however, the authors reported the use of laparoscopic endoscopic cooperative surgery (LECS), especially the use of non-exposure techniques, which might be challenging.

As the frequency of use of endoscopy increased, the occurrence of incidental GISTs increased as well. Endoscopic enucleation has been actively performed when GISTs are endophytically growing. This is because the method has advantages to

Received: March 2, 2018 Revised: March 13, 2018

Accepted: March 13, 2018

Correspondence: Chan-Young Kim

Division of Gastrointestinal Surgery, Department of Surgery, Chonbuk National University Medical School, 20 Gungi-ro, Deokjin-gu, Jeonju 54907, Korea Tel: +82-63-250-2298, Fax: +82-63-271-6197, E-mail: happyhill@jbnu.ac.kr ORCID: https://orcid.org/0000-0001-6656-9425

(c) This is an Open Access article distributed under the terms of the Creative Commons Attribution Non-Commercial License (http://creativecommons.org/ licenses/by-nc/3.0) which permits unrestricted non-commercial use, distribution, and reproduction in any medium, provided the original work is properly cited. surgery, such as lower cost and shorter hospital stay. However, points with low complete resection rate and high risk of perforation mask the advantage of endoscopic enucleation. Endoscopic full-thickness resection has also produced a successful result in skilled operators; however, it has the potential to cause peritoneal seeding problems. Finally, the successful and safe resection of endophytic GISTs, which are difficult to resect by endoscopy, requires the use of laparoscopy. Conversely, laparoscopic surgeons can remove simple exophytic GISTs effortlessly by using staplers. However, when the large intramural or large endophytic mass is removed by using a stapler, the seromuscular layer is excessively resected. This is likely to induce gastric stasis by overmodifying the shape of the stomach. In addition, the intramural mass near the esophagogastric junction or around the pyloric sphincter can be challenging to remove. Eventually, an endoscopist would be needed to prevent an excessive resection of the stomach wall and to avoid damage to the esophagogastric junction or pyloric sphincter. The first LECS was reported by Hiki et al. in 2008. ${ }^{4}$ Afterward, various methods developed with novel ideas were reported. The first to be reported was a simple method in which the endoscopist perforated the stomach wall by making a full-thickness incision around the mass by using a needle knife and then closing the wall with a laparoscopic procedure. However, this method caused the mass to be exposed to the abdominal cavity. Any Infection or spillage of tumor cells needed to be carefully considered and avoided. Therefore, the developed surgical method is the non-exposure surgery. 


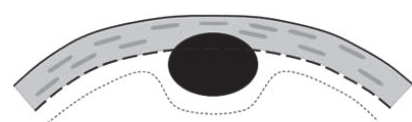

A

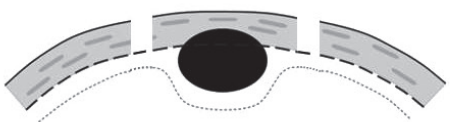

(B)

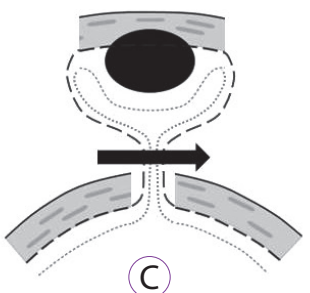

(C)

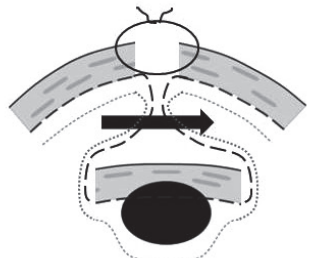

(D)

Fig. 1. Non-exposure endoscopic-laparoscopic cooperative surgery for stomach tumors. (A) Subepithelial tumor. (B) Seromuscular incision around the mass. (C) Removal of the mass with the combination of laparoscopic and endoscopic approaches to non-exposure technique procedure. (D) Removal of mass with the non-exposure endoscopic wall-inversion surgery procedure. (E) Figure in completion.

Fig. 1 shows a simplified drawing to help understand the two techniques used by the authors. Fig. $1 \mathrm{C}$ and D show the difference between CLEAN-NET and NEWS. CLEAN-NET (Fig. 1C) pushes the mass out of the stomach and removes the mass by using a laparoscopic stapler in the abdominal cavity. NEWS (Fig. 1D), on the other hand, inverts the mass into the stomach and is removed through the mouth by using an endoscope. The authors reported 12 cases of gastric tumor resection, including two patients with early gastric cancer; two using the CLEAN-NET technique, and the remaining using the NEWS technique. The authors reported successful operations without any major complications.

In conclusion, this paper suggests that LECS, including NEWS and CLEAN-NET, not only helps to preserve the patient's stomach function but also is selected based on the size, location, and direction of tumor growth. The challenging and successful results of this study show that advanced treatment of gastric subepithelial tumors (SETs) is feasible. Therefore, treating gastric SETs in a cooperative team between the surgeon and the endoscopist is an important requirement in the future.

Conflicts of Interest

The authors have no financial conflicts of interest.

\section{REFERENCES}

1. Hajer J, Havlůj L, Whitley A, Gürlich R. Non-exposure endoscopic-laparoscopic cooperative surgery for stomach tumors: first experience from the Czech Republic. Clin Endosc 2018;51:167-173.

2. Inoue $\mathrm{H}$, Ikeda H, Hosoya T, et al. Endoscopic mucosal resection, endoscopic submucosal dissection, and beyond: full-layer resection for gastric cancer with nonexposure technique (CLEAN-NET). Surg Oncol Clin N Am 2012;21:129-140.

3. Mitsui T, Niimi K, Yamashita H, et al. Non-exposed endoscopic wall-inversion surgery as a novel partial gastrectomy technique. Gastric Cancer 2014;17:594-599.

4. Hiki N, Yamamoto Y, Fukunaga T, et al. Laparoscopic and endoscopic cooperative surgery for gastrointestinal stromal tumor dissection. Surg Endosc 2008;22:1729-1735 